\title{
Association of excessive daytime sleepiness with migraine and headache frequency in the general population
}

\author{
Knut Stavem ${ }^{1,2,3,4^{*}} \mathbb{D}$, Håvard Anton Kristiansen ${ }^{1,2}$, Espen Saxhaug Kristoffersen ${ }^{1,5}$, Kari Jorunn Kværner ${ }^{1,6,7}$ \\ and Michael Bjørn Russell ${ }^{1,2}$
}

\begin{abstract}
Background: Some previous studies have postulated an association between migraine and excessive daytime sleepiness (EDS). This study evaluated the association of EDS with migraine and headache frequency in a general population, after adjusting for potential confounding variables.

Methods: The study was a postal survey of a random age and gender-stratified sample of 40,000 persons aged 20 to 80 years old drawn by the National Population Register in Norway. The questionnaire included questions about migraine, headache, the Epworth sleepiness scale (ESS) and various comorbidities. EDS was defined as ESS > 10. The association of EDS and migraine/headache were analysed by bivariate and multivariable logistic regression analyses.

Results: A total of 21,177 persons responded to the ESS and were included in the analyses. The odds ratio (OR) for EDS was increased for migraineurs $(1.42$ ( $95 \%$ Cl 1.31-1.54), $p<0.001)$ compared to non-migraineurs; however, this finding was not significant after adjustment for a number of possible confounders. EDS increased with increasing headache frequency, with an OR of 2.74 (95\% Cl 2.05-3.65), $p<0.001)$ for those with headache on $>179$ days per year compared to those without headache in multivariable analysis.
\end{abstract}

Conclusions: In a general population, the odds for EDS increased significantly with the headache frequency, irrespective of migraine status. EDS was not associated with reported migraine in multivariable analysis.

Keywords: Epidemiology, Excessive daytime sleepiness, Headache, Migraine

\section{Background}

Headache is the most common type of pain experienced by man, but receives little attention, possibly due to the often mild symptoms, and because most people rarely consult their physician due to headache [1]. Almost everyone experience tension-type headache once in a while, and migraine affects $10-20 \%$ of the general population [2, 3]. About $3 \%$ of the world population have chronic headache, i.e. mainly chronic tension-type headache or chronic migraine $[4,5]$.

Persons with headache and/or migraine often complain of sleepiness, a symptom with high clinical and public

\footnotetext{
* Correspondence: knut.stavem@medisin.uio.no

${ }^{1}$ Head and Neck Research Group, Research Centre, Akershus University

Hospital, Lørenskog, Norway

${ }^{2}$ Institute of Clinical Medicine, Campus Akershus University Hospital,

University of Oslo, Lørenskog, Norway

Full list of author information is available at the end of the article
}

health importance due to increased risk for accidents, decreased productivity and impaired quality of life [6-9]. Excessive daytime sleepiness (EDS) is experienced by $8-30 \%$ of the general population [10-13]. Despite the considerable burden of EDS, it is still under-reported, under-diagnosed and under-treated among those with chronic pain as well as in the general population [14-16].

Some clinic-based [17-20] or population-based [21] studies have proposed an association between migraine and EDS. Recently a large population-based study reported an association between migraine and EDS in bivariate analysis, but this association was weakened in multivariable analysis and became non-significant when adjusting for anxiety, depression, sleep duration and a score for sleep quality [22]. Moreover, whether EDS is associated with headache frequency in migraineurs varies between studies $[21,22]$. 
The present study aimed to investigate the association of EDS with migraine and headache frequency in an age and gender-stratified general population sample, after adjusting for possible confounders.

\section{Methods}

\section{Study design and sample}

An age and gender-stratified random sample of 40,000 persons aged 20-80 years old were drawn by the $\mathrm{Na}$ tional Population Register of Statistics Norway. Each of the ages $30,35,40,45,50,55$ and 60 years included 2,000 persons of each gender, while the remaining ages included 1,000 persons of each gender. The participants were residing in Akershus, Hedmark or Oppland County. The sample size was reduced to 38,871 because of error in the address list $(n=1,024)$, multi-handicap ( $n$ $=4)$, dementia $(n=23)$, insufficient Norwegian language skills $(n=3)$ and deceased $(n=75)$. The three counties have both rural and urban areas, and Akershus County is situated in close proximity to Oslo. Details of the study have previously been presented [23].

\section{Postal survey}

All participants received a mailed questionnaire with a standard letter containing information about the project. Apart from ensuring confidentiality and emphasising the importance of participation, it was stated that the objective was to study sleepiness during daytime. If the questionnaire evoked no response, a second mail was issued. The replies could either be on paper or web-based. The data were collected between February and August 2006. All questionnaires were scanned using TeleForm version 9 (Cardiff Software Ltd, Cambridge, UK).

\section{Migraine and headache}

The questions: "How many days during the last year have you had headache? (0 days, $1-11$ days, $12-30$ days, $31-84$ days, $85-179$ days or $\geq 180$ days)", and "Have you ever had migraine? (yes or no)" were used to screen for headache frequency and migraine. We applied the International Classification of Headache Disorders (ICHD-II) definitions slightly modified, i.e. no headache is 0 headache days within the last year, infrequent headache is $1-11$ headache days within the last year, frequent headache is $12-179$ headache days within the last year, while chronic headache is 180 days or more within the last year [24]. The term non-migraineurs describes those without migraine, independent of whether they reported headache or not.

\section{Daytime sleepiness and average sleep time}

Daytime sleepiness was assessed by the Epworth Sleepiness Scale (ESS). The ESS is a standardized questionnaire which describes eight daily situations in which the respondents estimate their likelihood of dozing off on a scale of 0 to 3 , i.e. $0=$ no chance of dozing, $1=$ slight chance of dozing, 2 = moderate chance of dozing and 3 = high chance of dozing [25]. The ESS score thereby ranges from 0 to 24 , and the results were dichotomized into scores $\leq 10$ and $>10$, the latter is considered to represent clinically significant EDS [26]. A question of average sleep time duration during the night was answered in hours and minutes and was categorized into three classes: short $(<6 \mathrm{~h})$, normal $(6-8 \mathrm{~h})$ and long $(>8 \mathrm{~h})$ sleep duration. In Norway, the ESS has exhibited properties in line with other language versions of the questionnaire [27].

\section{Smoking status and body mass index}

Smoking status was assessed with the item "How many cigarettes do you usually smoke per day?", coded as none, $1-10,11-20,>20$ for the analyses. Body mass index (BMI) in $\mathrm{kg} / \mathrm{m}^{2}$ was calculated from self-reported weight and height.

\section{Comorbidity}

To screen for depression, we asked the question: "Have you felt depressed or experienced diminished interest or pleasure in activities you usually enjoy?" This is a combination of two questions, where a "yes" answer to either of the two questions was considered a positive test [28]. The replies were dichotomized, i.e. never or rarely, $1-2$ times a month, $1-2$ times a week or $3-4$ times a week were classified as no depression, while almost daily was classified as depression.

The participants also responded to items about other comorbidities: previous myocardial infarction (yes or no), stroke (yes or no), current/previous angina pectoris (yes or no), asthma (yes or no), allergy (yes or no), hypertension (yes, no or unknown), or diabetes (yes or no). Finally, they responded to an item about ever having had treatment for a sleep disorder (yes or no).

\section{Statistical analyses}

Descriptive statistics are presented using the mean (SD) or number (\%). Groups were compared using the chisquare test.

Prevalence estimates are presented with cross-tables. To assess possible effect modification, the homogeneity of odds ratios (OR) for EDS in migraine vs. nonmigraine was tested in stratified analyses using the chisquare test.

The association of EDS with migraine and headache frequency was analysed by bivariate and multivariable logistic regression analyses with EDS $(\mathrm{ESS}>10=1$, ESS $\leq$ $10=0$ ) as the dependent variable. The six independent variables migraine, headache frequency, age, gender, BMI, and depression were included in the model. The 
modelling was conducted in several steps: (1) Bivariate models, (2) Multivariable models with the six variables mentioned above, (3) Multivariable models as (2) with the addition of the variables of allergy, angina pectoris, asthma, diabetes, hypertension, myocardial infarction and stroke, and (4) Multivariable models as (3) with the addition of the variables cigarette smoking, nightly sleep duration, and treatment for sleep disorder. Missing values were not imputed, and deletion of cases in the models was listwise.

Tests for multiplicative interactions were conducted between migraine*gender, headache frequency"gender, and migraine"headache frequency. Finally, model (3) was replicated in subsets of the respondents: non-migraineurs, migraineurs, men, and women.

All statistical analyses were performed using Stata version 14.1 (StataCorp, College Station, TX). We chose a $5 \%$ significance level, using two-sided tests.

\section{Ethical issues}

The project was approved by The Regional Committees for Medical Research Ethics and the Norwegian Social Science Data Services.

\section{Results}

The overall response rate was $54.5 \%(21,177 / 38,871)$. The respondents had a mean (SD) age of 50.5 (15.6) years and comprised $52.5 \%$ women (Table 1 ). Complete information on headache and migraine was reported by $89.4 \%$ of women and by $88.0 \%$ of men. Regarding replies to the ESS, $4.7 \%$ of women and $3.3 \%$ of men had missing values in one question, $0.7 \%$ of women and $0.6 \%$ of men had missing values in two questions, while $1.2 \%$ of women and $0.7 \%$ of men had missing values in three or more questions.

The 1-year prevalence of headache was $77.2 \%$; $84.0 \%$ $(8,872 / 10,558)$ in women and $69.6 \%(6,590 / 9,463)$ in men. The lifetime prevalence of migraine was $26.5 \%$; $34.1 \%(3,562 / 10,441)$ in women and $18.1 \%(1,696 / 9,392)$ in men $(p<0.001)$. In the total population the mean (SD) ESS score was 6.7 (4.0); among migraineurs 7.1 (4.2) $(n=5,258)$ and non-migraineurs 6.5 (3.9) $\quad(n=$ $14,575)$. The ESS scores according to migraine status (Fig. 1) and headache frequency (Fig. 2) showed skewed distributions in most subgroups.

Among migraineurs, non-migraineurs, and those with a missing response to the migraine item, the prevalence of EDS increased with headache frequency in all three groups (Table 2). The overall prevalence of EDS among the respondents $(n=21,177)$ was $17 \%$, and it was $20 \%$ among migraineurs, $15 \%$ among nonmigraineurs, and $19 \%$ among those with missing value on the migraine item.
Table 1 Descriptive statistics for survey respondents, number (\%) unless otherwise stated

\begin{tabular}{|c|c|c|}
\hline & $N$ & \\
\hline Age in years, mean (SD) & 21,177 & $50.5(15.6)$ \\
\hline Gender, women & 21,177 & $11,120(53)$ \\
\hline Epworth sleepiness scale score, mean (SD) & 21,177 & $6.7(4.0)$ \\
\hline Epworth sleepiness scale score >10 & 21,177 & $3,501(17)$ \\
\hline Migraine & 19,833 & $5,258(27)$ \\
\hline Headache frequency (days last year) & 20,021 & \\
\hline None & & $4,559(23)$ \\
\hline $1-11$ & & $8,109(41)$ \\
\hline $12-30$ & & $3,547(18)$ \\
\hline $31-84$ & & $2,280(11)$ \\
\hline 85-179 & & $951(5)$ \\
\hline 180 days or more & & $575(3)$ \\
\hline Body mass index in kg/m², mean (SD) & 20,818 & $25.8(4.2)$ \\
\hline \multicolumn{3}{|l|}{ Comorbidity } \\
\hline Symptoms of depression & 20,476 & $1,401(7)$ \\
\hline Myocardial infarction & 20,686 & $814(4)$ \\
\hline Stroke & 19,938 & $536(3)$ \\
\hline Angina pectoris & 20,681 & $978(5)$ \\
\hline Hypertension & 20,868 & \\
\hline No & & $14,903(71)$ \\
\hline Yes & & $3,591(17)$ \\
\hline Unkown & & $2,374(11)$ \\
\hline Diabetes & 20,685 & $934(5)$ \\
\hline Allergy & 20,121 & $6,564(33)$ \\
\hline Asthma & 19,156 & $2,287(12)$ \\
\hline Treated for sleep disorder & 20,749 & $1,251(6)$ \\
\hline Smoking (cigarettes per day) & 17,286 & \\
\hline None & & $11,902(69)$ \\
\hline $0-10$ & & $2,867(17)$ \\
\hline $11-20$ & & $2,252(13)$ \\
\hline$>20$ & & $265(2)$ \\
\hline Average nightly hours slept, mean (SD) & 20,907 & $7.0(1.1)$ \\
\hline \multicolumn{3}{|l|}{ Average nightly hours slept, categorized } \\
\hline$<6$ & & $1,750(8)$ \\
\hline $6-8$ & & $17,541(83)$ \\
\hline$>8$ & & $1,886(9)$ \\
\hline
\end{tabular}

There was no indication of heterogeneity of the ORs for EDS in migraine vs. non-migraine, or women vs. men in strata according to headache frequency (Table 3).

In bivariate logistic regression analysis, the odds ratio (OR) for having EDS was higher among migraineurs than non-migraineurs, and the OR for EDS increased with increasing headache frequency (Table 4). 


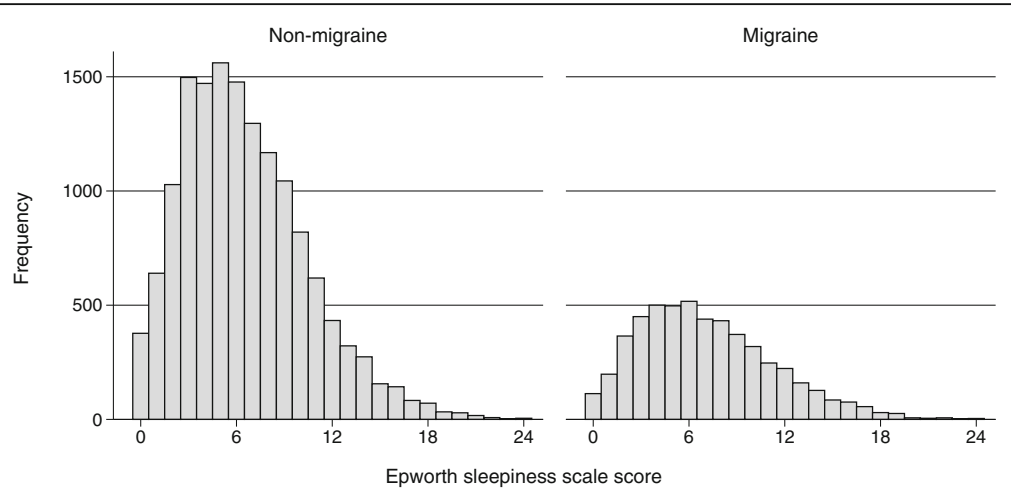

Fig. 1 Histogram of the frequency of Epworth sleepiness scale scores for participants according to migraine or non-migraine status

In multivariable logistic regression analysis, the relationship between migraine and EDS was weaker than in bivariate analysis, while the pattern of increasing OR for EDS with increasing headache frequency persisted in models after adjusting for age, BMI, depression, and gender (Table 4). Adjusting for additional potential comorbidities in two steps did not change this pattern (Table 4). Interaction terms between migraine"gender, headache frequency"gender, and migraine"headache were statistically not significant and were left out in the models.

In multivariable analysis in strata of the respondents, the pattern of ORs was consistent across migraineurs and non-migraineurs after adjusting for headache frequency, age, BMI, depression, gender and other comorbidities, and across women and men after adjusting for migraine, headache frequency, age, BMI, depression and other comorbidities (Table 5).

\section{Discussion}

This population-based cross-sectional study has demonstrated (1) a relationship between migraine and EDS in bivariate analysis, although this association was weakened and eventually disappeared in multivariable analysis, and (2) an association between headache frequency and EDS, which was consistent after adjustment for numerous potential confounders, and also when the analysis was replicated in subsets of the study population, i.e. among migraineurs, non-migraineurs, women or men.

Overall $17 \%$ met the criteria for having EDS, which is in line with that of a previous Norwegian telephone survey of a general population [29]. Our $20 \%$ prevalence of EDS in migraineurs was identical to that of Korean migraineurs from the general population [22]. The EDS prevalence in European clinic populations was 14\% in those with episodic migraine [18] and 20\% in chronic migraine [20], while it was substantial higher in an American clinic population, i.e. $32 \%$ in episodic migraineurs and $40 \%$ in chronic migraineurs [17]. The higher prevalence in the American study may partly be explained by a slightly higher mean BMI and lower ESS cut off, i.e. $\geq 10$ rather than $>10$ in the present and

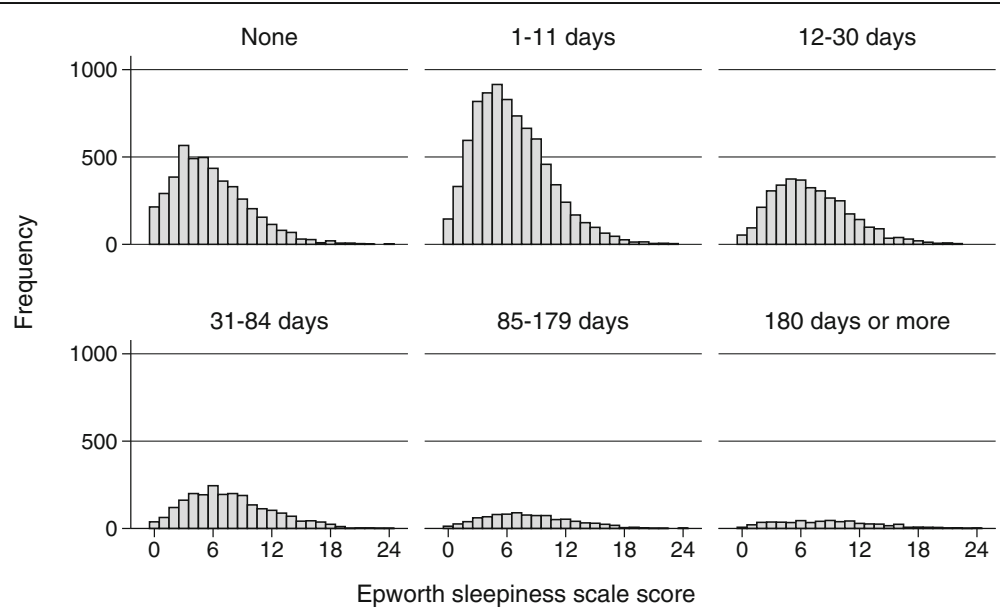

Fig. 2 Histogram of the frequency of Epworth sleepiness scale scores for participants according to categories of headache frequency (in number of days per month) 
Table 2 Prevalence of excessive daytime sleepiness (EDS), defined as Epworth sleepiness scale score $>10$, according to migraine and headache frequency

\begin{tabular}{|c|c|c|c|c|c|c|c|c|}
\hline \multirow[b]{2}{*}{ Headache frequency, days last year } & \multicolumn{2}{|c|}{ Non-migraine } & \multicolumn{2}{|c|}{ Migraine } & \multicolumn{2}{|c|}{ Missing migraine item } & \multicolumn{2}{|l|}{ Total } \\
\hline & $N$ & $n(\%)$ & $N$ & $n(\%)$ & $N$ & $n(\%)$ & $N$ & $n(\%)$ \\
\hline None & 3,814 & $413(11)$ & 390 & $56(14)$ & 355 & $56(16)$ & 4,559 & $525(12)$ \\
\hline $1-11$ & 6,159 & $862(14)$ & 1,568 & $228(15)$ & 382 & $59(15)$ & 8,109 & $1,149(14)$ \\
\hline $12-30$ & 2,040 & $378(19)$ & 1,302 & $238(18)$ & 205 & $41(20)$ & 3,547 & 657 (19) \\
\hline $31-84$ & 1,019 & $227(22)$ & 1,087 & $271(25)$ & 174 & $42(24)$ & 2,280 & $540(24)$ \\
\hline 85-179 & 411 & $115(28)$ & 482 & $139(29)$ & 58 & $14(24)$ & 951 & $268(28)$ \\
\hline$\geq 180$ & 219 & $76(35)$ & 294 & $102(35)$ & 62 & $22(35)$ & 575 & $200(35)$ \\
\hline Missing & 913 & $124(14)$ & 135 & $22(16)$ & 108 & $15(14)$ & 1,156 & $162(14)$ \\
\hline Total & 14,575 & $2,196(15)$ & 5,258 & $1,056(20)$ & 1,236 & 234 (19) & 21,177 & $3,501(17)$ \\
\hline
\end{tabular}

Korean studies. The European clinic studies included 100 person with episodic and chronic migraine, respectively, while the American clinic study included 72 persons with episodic migraine and 128 persons with chronic migraine, providing relative broad confidence intervals for the EDS prevalence, i.e. 14\% (8-22\%), 20\% (13-29\%), 32\% (25-48\%) and 40\% (31-49\%).

The present study found an association between migraine and EDS in bivariate analysis, which was weaker in the multivariable analyses and disappeared when adjusting for all potential confounders, a finding similar to that of The Korean population-based study when adjusting for anxiety, depression, sleep duration and Pittsburgh Sleep Quality index score [22]. These findings are in concert with another population-based study reporting increased odds of EDS in migraineurs compared with headache-free individuals after adjustment for age, sex and sleep-promoting medication [21]. The findings are also supported by a case-control study of episodic migraine $[18,20]$, as well as a study including 370 migraineurs and 119 non-migraineurs [20]. The latter study found a statistically significant association of chronic migraine and EDS with an OR of 3.9 (95\% CI 1.5.-10.2), although the mean ESS score was not significant different between those with chronic migraine and healthy controls [20].

In contrast, one study reported a statistically significant association between migraine and sleep problems after adjusting for both lifetime and current anxiety and mood disorders [30]. This was a controlled study using a systematic assessment of migraine, sleep problems and anxiety and mood disorders with interviews, in contrast to the present, larger questionnaire-based survey.

In the present study, the prevalence of EDS increased with headache frequency, independent of migraine status, in line with a previous general population study [21], although in the latter study the increased ORs for EDS with increasing headache frequency were statistically not significant. Therefore, it is possibly the frequency of the pain rather than migraine or tension-type headache in itself that is associated with EDS.

There was no evidence of effect modification of gender or headache frequency on the association of migraine with EDS, or of gender on the association of headache frequency with EDS, as indicated by the homogeneity of the ORs and the lack of significant interactions in the regression models.

Table 3 Odds ratios and 95\% confidence intervals (95\% Cl) for having excessive daytime sleepiness (EDS) for migraineurs compared to non-migraineurs, and men versus women. Stratified analyses according to headache frequency categories

\begin{tabular}{|c|c|c|c|c|}
\hline \multirow[b]{2}{*}{ Headache frequency, days last year } & \multicolumn{2}{|c|}{ Migraine vs. non-migraine } & \multicolumn{2}{|l|}{ Men vs. women } \\
\hline & Odds ratio $(95 \% \mathrm{Cl})$ & P & Odds ratio $(95 \% \mathrm{Cl})$ & $P$ \\
\hline None & $1.38(1.02-1.87)$ & 0.035 & $1.25(1.03-1.51)$ & 0.026 \\
\hline $1-11$ & $1.05(0.89-1.22)$ & 0.58 & $1.35(1.19-1.53)$ & $<0.001$ \\
\hline $12-30$ & $0.98(0.82-1.18)$ & 0.86 & $1.34(1.13-1.59)$ & $<0.001$ \\
\hline $31-84$ & $1.16(0.95-1.42)$ & 0.152 & $1.36(1.11-1.66)$ & 0.003 \\
\hline $85-179$ & $1.04(0.78-1.40)$ & 0.78 & $1.21(0.90-1.64)$ & 0.21 \\
\hline$\geq 180$ & $1.00(0.69-1.44)$ & 1.00 & $0.91(0.63-1.32)$ & 0.62 \\
\hline Missing & $1.23(0.75-2.01)$ & 0.42 & $1.37(0.98-1.91)$ & 0.068 \\
\hline Test of homogeneity of odds ratios: & $\operatorname{Chi}^{2}(6)=4.69, P=0.58$ & & $\mathrm{Chi}^{2}(6)=4.73, P=0.58$ & \\
\hline
\end{tabular}


Table 4 Odds ratios with 95\% confidence intervals (95\%Cl) for having excessive daytime sleepiness (EDS), logistic regression analysis

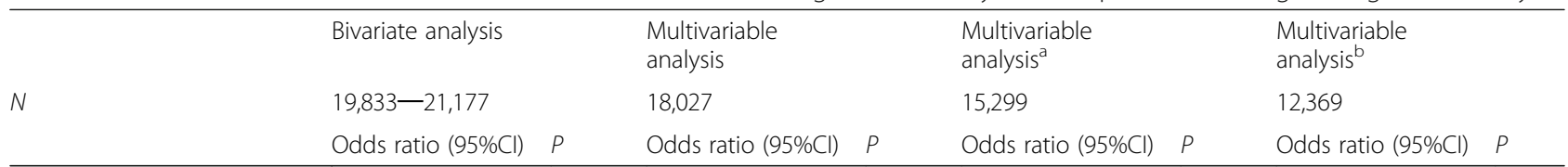

\section{Migraine}

\section{No}

Yes

Headache frequency, days per year

$$
\begin{aligned}
& \text { None } \\
& 1-11 \\
& 12-30 \\
& 31-84 \\
& 85-179 \\
& \geq 180
\end{aligned}
$$

Age, per 10 years

Gender

Women

Body mass index, per $\mathrm{kg} / \mathrm{m} 2$

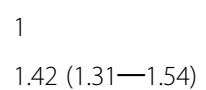

$$
\begin{gathered}
1 \\
<0.001 \quad 1.11(1.01-1.22)
\end{gathered}
$$$$
0.032
$$$$
1.11(1.00-1.23)
$$$$
0.044
$$$$
1.08(0.96-1.21)
$$

0.196

\section{year}

$27(1.14--1.42)$

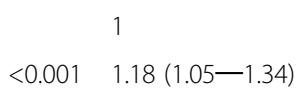

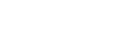

$.75(1.54-1.98)$

$<0.001 \quad 1.54(1.33-1.78)$

0.009

1

1

$<0.001 \quad 1.53(1.31-1.80)$

0.003

1.17

$<0.001 \quad 1.50(1.25-1.79)$

$<0.001 \quad 2.00(1.65-2.43)$

$<0.001 \quad 2.26(1.77-2.87)$

$<0.001 \quad 2.74(2.05-3.65)$

$<0.001 \quad 0.88(0.84-0.91)$

0.05

$<0.001 \quad 2.02(1.70-2.40)$

$<0.001 \quad 2.39(1.93-2.96)$

$<0.001 \quad 3.12(2.42-4.02)$

$<0.001 \quad 0.88(0.85-0.91)$

\begin{tabular}{|c|c|c|c|c|c|c|c|c|}
\hline No & 1 & & 1 & & 1 & & 1 & \\
\hline Yes & $2.99(2.66-3.36)$ & $<0.001$ & $2.45(2.15-2.80)$ & $<0.001$ & $2.48(2.13-2.88)$ & $<0.001$ & $2.20(1.86-2.62)$ & $<0.001$ \\
\hline
\end{tabular}

$<0.001$

$<0.001$

$<0.001$

$<0.001$

$<0.001$

Symptoms of depression

adjusted for additional potential confounders allergy, angina pectoris, asthma, diabetes, hypertension, myocardial infarction and stroke

${ }^{b}$ adjusted for additional potential confounders (as above) + cigarette smoking $(0,1-10,11-20,>20$ per day), nightly sleep $(<6 \mathrm{~h}, 6-8 \mathrm{~h},>8 \mathrm{~h})$ and previous treatment for sleep disorder

This study had several strengths. Firstly, it was a very large population-based survey. Secondly, the multivariable analyses were conducted in three consecutive steps and adjusted for an increasing number of possible confounders in each step.

Several potential limitations of this study should be mentioned. The response rate was relatively low; however, it was comparable to that of some other sleep-related epidemiologic studies [29, 31] and higher than in recent population-based studies of headache and sleep [21, 22].

The present study defined EDS as ESS $>10$, in line with the recommendation of the developer of the ESS [32], recent epidemiological studies [33-35] and a recent population-based study of headache and sleep [22]. In contrast, for unknown reasons, most previous studies of the association of migraine or headache frequency with EDS defined EDS as ESS $\geq 10$ [17-21], which would lead to a higher prevalence of EDS. The ESS is widely used in evaluating subjective daytime sleepiness $[25,36]$. However, the ESS has been criticized because it shows little association with objective measures, such as the multiple sleep latency test (MSLT) [37-39]. The large number of participants in this study precluded the use of MSLT for more comprehensive assessment of daytime sleepiness.
Another potential limitation is that the data reported in the present study were self-reported. This study asked about lifetime prevalence of migraine. The single question about lifetime occurrence of migraine, which was used, has previously been evaluated against a clinical interview by two physicians in another Norwegian survey in eastern Akershus County, reporting a raw agreement rate of 0.81 and kappa ( $\kappa$ ) of 0.62 , which is considered to be a good agreement [23]. Two previous epidemiological surveys used the same screening question for migraine as in the present study, and the observed agreement rates were 0.92 and 0.94 , and $\kappa$ were 0.77 and 0.81 , respectively $[2,40]$. Due to the high number of participants in our survey, it was not feasible to apply the gold standard, i.e. a clinical interview by a physician/neurologist with expertise in headache diagnostics. The current study did not investigate the effect of EDS on clinical characteristics of migraine, which would require more detailed clinical information than is possible in a questionnaire-based general population survey.

Single questions about tension-type headache and its frequency have been compared with a clinical interview, and the agreement was good $[3,40]$. We think our 
Table 5 Odds ratios and 95\% confidence intervals (95\% Cl) for having excessive daytime sleepiness (EDS), multivariable logistic regression analysis ${ }^{\mathrm{a}}$ in strata of the population

\begin{tabular}{|c|c|c|c|c|c|c|c|c|}
\hline \multirow{3}{*}{ N } & \multicolumn{2}{|l|}{ Migraineurs } & \multicolumn{2}{|l|}{ Non-migraineurs } & \multicolumn{2}{|l|}{ Men } & \multicolumn{2}{|l|}{ Women } \\
\hline & \multicolumn{2}{|l|}{4,126} & \multicolumn{2}{|l|}{11,173} & \multicolumn{2}{|l|}{7,313} & \multicolumn{2}{|l|}{7,986} \\
\hline & Odds ratio $(95 \% \mathrm{Cl})$ & $P$ & Odds ratio $(95 \% \mathrm{Cl})$ & $P$ & Odds ratio $(95 \% \mathrm{Cl})$ & $P$ & Odds ratio $(95 \% \mathrm{Cl})$ & $P$ \\
\hline \multicolumn{9}{|l|}{ Migraine } \\
\hline No & - & & - & & 1 & & 1 & \\
\hline Yes & - & & - & & $1.18(1.01-1.38)$ & 0.041 & $1.07(0.94-1.22)$ & 0.31 \\
\hline \multicolumn{9}{|c|}{ Headache frequency, days per year } \\
\hline None & 1 & & 1 & & 1 & & & \\
\hline $1-11$ & $0.93(0.63-1.37)$ & 0.73 & $1.27(1.09-1.47)$ & 0.002 & $1.32(1.11-1.57)$ & 0.001 & $1.09(0.86-1.37)$ & 0.48 \\
\hline $12-30$ & $1.21(0.82-1.79)$ & 0.34 & $1.58(1.32-1.89)$ & $<0.001$ & $1.63(1.31-2.03)$ & $<0.001$ & $1.37(1.07-1.75)$ & 0.013 \\
\hline $31-84$ & $1.74(1.17-2.57)$ & 0.006 & $1.92(1.55-2.37)$ & $<0.001$ & $2.30(1.79-2.94)$ & $<0.001$ & $1.74(1.34-2.25)$ & $<0.001$ \\
\hline $85-179$ & $1.90(1.24-2.91)$ & 0.003 & $2.52(1.91-3.34)$ & $<0.001$ & $2.33(1.65-3.30)$ & $<0.001$ & $2.21(1.64-2.96)$ & $<0.001$ \\
\hline$\geq 180$ & $2.72(1.71-4.30)$ & $<0.001$ & $2.92(2.04-4.19)$ & $<0.001$ & $2.43(1.57-3.77)$ & $<0.001$ & $3.21(2.30-4.50)$ & $<0.001$ \\
\hline Age, per 10 years & $0.90(0.84-0.95)$ & 0.001 & $0.87(0.84-0.91)$ & $<0.001$ & $0.92(0.88-0.97)$ & 0.001 & $0.85(0.81-0.89)$ & $<0.001$ \\
\hline \multicolumn{9}{|l|}{ Sex } \\
\hline Women & 1 & & 1 & & - & & - & \\
\hline Men & $1.31(1.11-1.54)$ & 0.002 & $1.18(1.06-1.32)$ & 0.003 & - & & - & \\
\hline Body mass index, per $\mathrm{kg} / \mathrm{m}^{2}$ & $1.04(1.02-1.05)$ & $<0.001$ & $1.04(1.03-1.06)$ & $<0.001$ & $1.05(1.03-1.06)$ & $<0.001$ & $1.04(1.03-1.05)$ & $<0.001$ \\
\hline \multicolumn{9}{|l|}{ Symptoms of depression } \\
\hline No & 1 & & 1 & & 1 & & & \\
\hline Yes & $2.18(1.70-2.79)$ & $<0.001$ & $2.66(2.21-3.21)$ & $<0.001$ & $2.57(2.05-3.22)$ & $<0.001$ & $2.43(1.99-2.97)$ & $<0.001$ \\
\hline
\end{tabular}

adjusted for additional potential confounders allergy, angina pectoris, asthma, diabetes, hypertension, myocardial infarction and stroke

question regarding headache was at least as precise as the single question about tension-type headache, because the term headache is known by everybody, while some subjects may not know what tension-type headache is. Similarly, we think the frequency report on headache is likely to be precise.

The data on headache frequency was based on headache during the last year, which is likely to be less subject to recall bias as compared to headache earlier in life.

Moreover, the single question about self-reported depression might be less precise than a more extensive questionnaire or diagnostic interview. However, the combination of the two questions into one item has been shown to have a sensitivity of $96 \%(95 \% \mathrm{CI}$ $90-99 \%)$ and specificity of $57 \%$ (95\% CI $53-62 \%)$ in relation to major depression [28]. This compares with a sensitivity of $89-96 \%$ and specificity $51-72 \%$, when ascertained with more extensive questionnaires [28].

The present study did not assess the quality of sleep, or the use of analgesics that may be associated with headaches and EDS, or $\beta$-blockers that may be used in prophylactic treatment of migraine and may be associated with EDS. However, only a small minority of migraineurs use $\beta$-blockers.

The focus of this paper was on the association of migraine and headache frequency with EDS, and not on a general prediction model for EDS. Therefore, we have focused on factors that may confound the association of migraine and/or headache frequency with EDS, and on possible effect modification between some of these variables. EDS depends on several factors known to interfere with the sleep-awake cycle, while headache and migraine are mainly known to vary with gender. For example, caffeine, alcohol, employment including nightshifts, restless legs syndrome, concomitant urological diseases, menstruation and thyroid hormones may be associated with EDS. However, based on the literature, we are not aware of firm evidence for an association of these factors with migraine or tension-type headache, and if such an association exists it is most likely of limited importance.

\section{Conclusions}

This study showed that EDS was associated with an increase in headache frequency in both migraineurs and non-migraineurs. In contrast, the association between migraine and EDS disappeared after adjustment for a number of confounders.

\section{Abbreviations}

EDS: Excessive daytime sleepiness; ESS: Epworth sleepiness scale; ICHD: International classification of headache disorders; MSLT: Multiple sleep latency test; SD: Standard deviation 


\section{Acknowledgements}

None.

\section{Funding}

This study was supported by a grant from South-East Norway Regional Health Authority, grant number 2004219.

\section{Availability of data and materials}

The data that support the findings of this study are available on request from the corresponding author [KS]. The data are not publicly available because of information that could compromise research participant privacy.

\section{Authors' contributions}

KJK and MBR designed the project. HAK contributed to planning, data collection, and data cleaning. KS conducted the analysis with support from MBR. KS, HAK and MBR drafted the manuscript. All authors contributed to the manuscript with critical revisions and suggestions. All authors approved the final manuscript

\section{Competing interests}

The authors declare that they have no competing interests.

\section{Consent for publication}

Not applicable.

\section{Ethics approval and consent to participate}

The project was approved by The Regional Committees for Medical Research Ethics and the Norwegian Social Science Data Services.

\section{Publisher's Note}

Springer Nature remains neutral with regard to jurisdictional claims in published maps and institutional affiliations.

\section{Author details}

'Head and Neck Research Group, Research Centre, Akershus University Hospital, Lørenskog, Norway. ${ }^{2}$ Institute of Clinical Medicine, Campus Akershus University Hospital, University of Oslo, Lørenskog, Norway. ${ }^{3}$ Department of Pulmonary Medicine, Medical Division, Akershus University Hospital, Lørenskog, Norway. ${ }^{4}$ Health Services Research Unit, Akershus University Hospital, Lørenskog, Norway. ${ }^{5}$ Department of General Practice, University of Oslo, Oslo, Norway. ${ }^{6} \mathrm{C} 3$ Centre for Connected Care, Oslo University Hospital, Oslo, Norway. ${ }^{7}$ BI Norwegian Business School, Oslo, Norway.

\section{Received: 9 February 2017 Accepted: 7 March 2017}

\section{Published online: 20 March 2017}

\section{References}

1. Lyngberg AC, Rasmussen BK, Jorgensen T, Jensen R (2005) Secular changes in health care utilization and work absence for migraine and tension-type headache: a population based study. Eur J Epidemiol 20(12):1007-1014

2. Russell MB, Rasmussen BK, Thorvaldsen P, Olesen J (1995) Prevalence and sex-ratio of the subtypes of migraine. Int J Epidemiol 24(3):612-618

3. Russell MB, Levi N, Saltyte-Benth J, Fenger K (2006) Tension-type headache in adolescents and adults: a population based study of 33,764 twins. Eur J Epidemiol 21(2):153-160

4. Jensen R, Stovner LJ (2008) Epidemiology and comorbidity of headache. Lancet Neurol 7(4):354-361

5. Grande RB, Aaseth K, Gulbrandsen P, Lundqvist C, Russell MB (2008) Prevalence of primary chronic headache in a population-based sample of 30- to 44-year-old persons. The Akershus study of chronic headache. Neuroepidemiology 30(2):76-83

6. Mitler MM, Carskadon MA, Czeisler CA, Dement WC, Dinges DF, Graeber RC (1988) Catastrophes, sleep, and public policy: consensus report. Sleep 11(1): 100-109

7. Roth T, Roehrs TA (1996) Etiologies and sequelae of excessive daytime sleepiness. Clin Ther 18(4):562-576, discussion 561

8. Leger D (1994) The cost of sleep-related accidents: a report for the national commission on sleep disorders research. Sleep 17(1):84-93

9. Briones B, Adams N, Strauss M, Rosenberg C, Whalen C, Carskadon M, Roebuck T, Winters M, Redline S (1996) Relationship between sleepiness and general health status. Sleep 19(7):583-588
10. Young T, Palta M, Dempsey J, Skatrud J, Weber S, Badr S (1993) The occurrence of sleep-disordered breathing among middle-aged adults. N Engl J Med 328(17):1230-1235

11. Duran J, Esnaola S, Rubio R, Iztueta A (2001) Obstructive sleep apneahypopnea and related clinical features in a population-based sample of subjects aged 30 to 70 yr. Am J Respir Crit Care Med 163(3 Pt 1):685-689

12. Bixler EO, Vgontzas AN, Lin HM, Calhoun SL, Vela-Bueno A, Kales A (2005) Excessive daytime sleepiness in a general population sample: the role of sleep apnea, age, obesity, diabetes, and depression. J Clin Endocrinol Metab 90(8):4510-4515

13. Stradling JR, Barbour C, Glennon J, Langford BA, Crosby JH (2000) Prevalence of sleepiness and its relation to autonomic evidence of arousals and increased inspiratory effort in a community based population of men and women. J Sleep Res 9(4):381-388

14. Stiefel F, Stagno D (2004) Management of insomnia in patients with chronic pain conditions. CNS Drugs 18(5):285-296

15. Young TB (2004) Epidemiology of daytime sleepiness: definitions, symptomatology, and prevalence. J Clin Psychiatry 65(Suppl 16):12-16

16. Leger D, Poursain B, Neubauer D, Uchiyama M (2008) An international survey of sleeping problems in the general population. Curr Med Res Opin 24(1):307-317

17. Peres MF, Stiles MA, Siow HC, Silberstein SD (2005) Excessive daytime sleepiness in migraine patients. J Neurol Neurosurg Psychiatry 76(10):14671468

18. Barbanti P, Fabbrini G, Aurilia C, Vanacore N, Cruccu G (2007) A case-control study on excessive daytime sleepiness in episodic migraine. Cephalalgia 27(10):1115-1119

19. Seidel S, Hartl T, Weber M, Matterey S, Paul A, Riederer F, Gharabaghi M, Wober-Bingol C, Wober C (2009) Quality of sleep, fatigue and daytime sleepiness in migraine - a controlled study. Cephalalgia 29(6):662-669

20. Barbanti P, Aurilia C, Egeo G, Fofi L, Vanacore N (2013) A case-control study on excessive daytime sleepiness in chronic migraine. Sleep Med 14(3):278-281

21. Ødegård SS, Engstrøm M, Sand T, Stovner L, Zwart JA, Hagen K (2010) Associations between sleep disturbance and primary headaches: the third Nord-Trondelag Health Study. J Headache Pain 11(3):197-206

22. Kim J, Cho SJ, Kim WJ, Yang KI, Yun CH, Chu MK (2016) Excessive daytime sleepiness is associated with an exacerbation of migraine: A populationbased study. J Headache Pain 17(1):62

23. Russell MB, Kristiansen HA, Saltyte-Benth J, Kvaerner KJ (2008) A crosssectional population-based survey of migraine and headache in 21,177 Norwegians: the Akershus sleep apnea project. J Headache Pain 9(6): 339-347

24. Society HCSotIH (2004) The International Classification of Headache Disorders: 2nd edition. Cephalalgia 24(Suppl 1):9-160

25. Johns MW (1991) A new method for measuring daytime sleepiness: the Epworth sleepiness scale. Sleep 14(6):540-545

26. Johns MW (1994) Sleepiness in different situations measured by the Epworth Sleepiness Scale. Sleep 17(8):703-710

27. Beiske KK, Kjelsberg FN, Ruud EA, Stavem K (2009) Reliability and validity of a Norwegian version of the Epworth sleepiness scale. Sleep Breath 13(1):65-72

28. Whooley MA, Avins AL, Miranda J, Browner WS (1997) Case-finding instruments for depression. Two questions are as good as many. J Gen Intern Med 12(7):439-445

29. Pallesen S, Nordhus IH, Omvik S, Sivertsen B, Tell GS, Bjorvatn B (2007) Prevalence and risk factors of subjective sleepiness in the general adult population. Sleep 30(5):619-624

30. Vgontzas A, Cui L, Merikangas KR (2008) Are sleep difficulties associated with migraine attributable to anxiety and depression? Headache 48(10): 1451-1459

31. Ancoli-Israel S, Roth T (1999) Characteristics of insomnia in the united states: results of the 1991 national sleep foundation survey. I. Sleep 22(Suppl 2): S347-353

32. Johns MW, Hocking B (1997) Daytime sleepiness and sleep habits of Australian workers. Sleep 20(10):844-849

33. Gander PH, Marshall NS, Harris R, Reid P (2005) The Epworth sleepiness scale: influence of age, ethnicity, and socioeconomic deprivation. Epworth sleepiness scores of adults in New Zealand. Sleep 28(2):249-253

34. Sanford SD, Lichstein KL, Durrence HH, Riedel BW, Taylor DJ, Bush AJ (2006) The influence of age, gender, ethnicity, and insomnia on Epworth sleepiness scores: a normative US population. Sleep Med 7(4):319-326 
35. Sander C, Hegerl U, Wirkner K, Walter N, Kocalevent RD, Petrowski K, Glaesmer H, Hinz A (2016) Normative values of the Epworth sleepiness scale (ESS), derived from a large German sample. Sleep Breath 20(4):1337-1345

36. Johns MW (1992) Reliability and factor analysis of the Epworth sleepiness scale. Sleep 15(4):376-381

37. Olson LG, Cole MF, Ambrogetti A (1998) Correlations among Epworth sleepiness scale scores, multiple sleep latency tests and psychological symptoms. J Sleep Res 7(4):248-253

38. Chervin RD, Aldrich MS (1999) The Epworth Sleepiness Scale may not reflect objective measures of sleepiness or sleep apnea. Neurology 52(1):125-131

39. Furuta H, Kaneda R, Kosaka K, Arai H, Sano J, Koshino Y (1999) Epworth sleepiness scale and sleep studies in patients with obstructive sleep apnea syndrome. Psychiatry Clin Neurosci 53(2):301-302

40. Rasmussen BK, Jensen R, Olesen J (1991) Questionnaire versus clinical interview in the diagnosis of headache. Headache 31:290-295

\section{Submit your manuscript to a SpringerOpen ${ }^{\circ}$ journal and benefit from:}

- Convenient online submission

- Rigorous peer review

- Immediate publication on acceptance

- Open access: articles freely available online

- High visibility within the field

- Retaining the copyright to your article

Submit your next manuscript at $>$ springeropen.com 